\title{
Comparing the Performance of Different Classifiers for Posture Detection
}

\author{
Sagar Suresh Kumar ${ }^{1}$, Kia Dashtipour ${ }^{1,2}$, Mandar Gogate ${ }^{2}$, Jawad Ahmad ${ }^{2}$, \\ Khaled Assaleh ${ }^{3}$, Kamran Arshad ${ }^{3}$, Muhammad Ali Imran ${ }^{1,4}$, and Qammer \\ Abbai $^{1}$ Wasim Ahmad ${ }^{1}$ \\ 1. James Watt School of Engineering, University of Glasgow,Glasgow, United \\ Kingdom \\ 2. School of Computing, Edinburgh Napier University, Edinburgh, U.K. \\ 3. Faculty of Engineering and IT, Ajman University, Ajman 346, UAE \\ 4. Artificial Intelligence Research Center (AIRC), Ajman University, Ajman, UAE
}

\begin{abstract}
Human Posture Classification(HPC) is used in many fields such as as human computer interfacing, security surveillance, rehabilitation, remote monitoring, and so on. This paper compares the performance of different classifiers in the detection of 3 postures, sitting, standing, and lying down, which was recorded using Microsoft Kinect cameras. The Machine Learning classifiers used included the Support Vector Classifier, Naive Bayes, Logistic Regression, K-Nearest Neighbours, and Random Forests. The Deep Learning ones included the standard Multi-Layer Perceptron, Convolutional Neural Networks(CNN), and Long Short Term Memory Networks(LSTM). It was observed that Deep Learning methods outperformed the former and that the one-dimensional CNN performed the best with an accuracy of $93.45 \%$.
\end{abstract}

Keywords: Machine learning · Deep learning · Detecting Alzheimer.

\section{Introduction}

Human Posture Classification(HPC) has applications in a wide range of fields such as human computer interfacing, security surveillance, rehabilitation, remote monitoring, and so on 20]. There are broadly two kinds of sensor schemes that can be used for HPC: wearable and contactless. Wearable methods use sensors such as accelerometers, gyroscopes, magnetometers or even sensors that use physiological data such as Electromyogram(EMG), on different locations of the body while contactless ones rely on image or video processing, and also sensors in the proximity of the patient. Generally, wearable methods are obtrusive and cause discomfort. This is especially true for the elderly and the physically compromised. Hence, the research area of contactless HPC has gained popularity in the past few years $4,7,7,11,19,26,40,41,45]$.

HPC has been used to identify and correct several medical ailments in the past. Matar et al. used contactless bed-sheet pressure sensors to detect bedposture and prevent pressure ulcers [38. Lee et al. used inertial sensors to 
monitor squat exercises to prevent knee and leg injuries 33. In camera based methods, Microsoft Kinect devices, which provides depth images using a combination of RGB and IR cameras and outputs the 3D coordinates of 32 joints, are quite popular. These solutions have benefited from the developments Machine and Deep Learning [3. However, most of the past papers just use one or two classifiers without addressing in detail the classification schemes that produce optimal results. Hence, this work extensively compares the performance of different machine and deep learning classifiers and attempts to ascertain the optimum classifier(s) for posture data.

The rest of the paper is structured as follows. Section 2 provides the background information related to posture detection. Section 3 presents the proposed methodology. Section 4 provides the experimental results of the proposed posture detection and discussion, and finally Section 5 concludes the paper.

\section{Related Work}

Sidrah et al. 34 developed a novel approach based on hybrid approach used machine learning and deep learning approaches to detect human posture detection. However, the proposed approach can detect falling and standing and it cannot detect multiple posture detection. Panini et al. 42 presented an approach based on posture human detection in domotic application. The machine learning is used to generate the probability maps. The statistic classifier used to compare the probability maps and histogram profiles extracted from moving of people. The experimental results show that the results are robust and computational time are lowers as compared to state-of-the-art approaches such

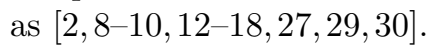

Ma et al. 37 proposed a cushion-based posture detection system used to process sensor for human detection in the wheelchair. The method is consists of three different steps such as classification for posture, backward selection of sensor configuration, and comparison with state-of-the-art approaches 1, 5, 6, \begin{tabular}{l|l|l|l|l|l|l|}
21 & 25 & 28 & 31 & 32 & 35 & $36,46,47$.
\end{tabular}

Nasirahmadi et al. [39] proposed an approach to identify whether a twodimensional imaging system along with deep learning approaches to detecting standing and lying postures with CNN and ResNet features extraction of RGB images were used. Sacchetti et al. [44] developed an approach to classify human posture detection in classroom ambience. The posture can be divided into confident and non confident aiming for teacher evaluation, interested/non interested. The approach presents some concepts about postures and how effectively detect

openpose library and finally neural network is used measure the effectiveness of the approach.

\section{Methodology}

In this section, we discussed the proposed methodology for human posture detection. The Fig 1 displays the overview framework of the approach. 


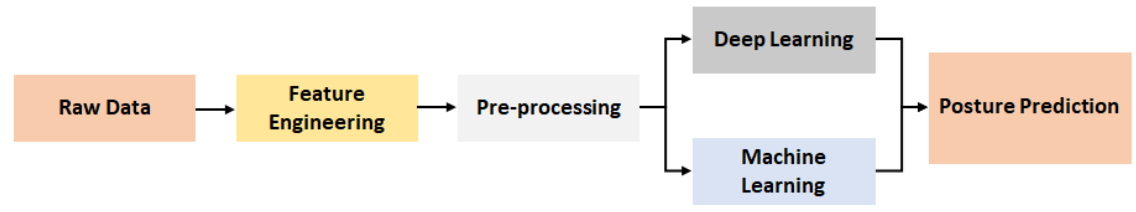

Fig. 1. Overview Proposed Approach

\subsection{Classifiers}

A wide range of machine and deep learning classifiers were used and are illustrated in Table 1 and Table 2. The Waikato Environment for Knowledge Analysis (WEKA) platform was used in this study to implement these algorithms. Among machine learning algorithms, the Naive Bayes Classifier's prior probabilities were set to 0, the Logistic Regression model used L2 loss, the K-nearest neighbours algorithm used 30 neighbours, and the the Random Forests classifier used 100 decision trees.

Convolutional Neural Network: For comparison, the novel CNN framework is developed. The implemented CNN consists of input, hidden and output layers. Our proposed CNN framework contains convolutional, max pooling and fully connected layers. The 10-layered CNN framework achieved the most promising results. The Fig 2 shows the architecture for CNN.

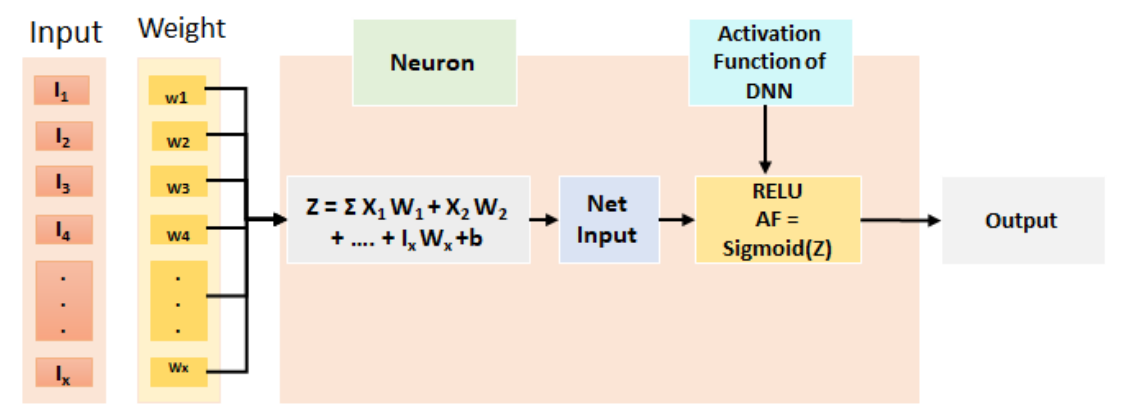

Fig. 2. Overview architecture of CNN

Long Short Term Memory (LSTM): The long short term memory (LSTM) proposed architecture contains input layer, two different stacked LSTM and one output as fully connected layer. Particularly, the LSTM architecture consists of 
two different stacked bidirectional layers (contains 128 cells and 64 cells) with dropout 0.2 and a dense layer with two neurons and softmax activation.

Among deep learning algorithms, both 1D and 2D Convolutional Neural Networks were used and their structures consisting of max pooling, convolution, and fully connected layers ( total 10) are illustrated. Both unidirectional and bidirectional Long Short Term Memory(LSTM) Networks were used consisting of 2 stacked layers of 128 and 64 cells and a dense layer of softmax activation function. The Multilayer Perceptron(MLP) consisted of 2 hidden layers and used the $\mathrm{ReLu}$ activation function. $\mathrm{K}$ fold cross validation was used and $\mathrm{K}$ values of 10,5 , and 2 were tested.

\section{Results and Discussion}

The Deep Learning based classifiers have outperformed the Machine Learning Classifiers in general, as shown in Table 17and 2. As expected, as the number of K-folds decreased, the accuracies decreased as well. Among Machine Learning methods, the Support Vector Machine with Radial Basis Function(RBF) Kernel performed the best with an overall accuracy of $83.42 \%$ while the Naive Bayes performed the worst with an accuracy of $74.56 \%$. In Deep Learning methods, CNNs and LSTMs were superior to the traditional MLP networks. Although the latter 2 had similar accuracies, the 1D-CNN performed the best with an accuracy of $93.45 \%$. Thus, future work should focus on optimizing the structure and performance of this network in posture detection.

Dataset: The data from [43] was used in this study, in which Microsoft Kinect V2 cameras were used to extract posture information of six users and three activities: sitting, standing, and lying down. Although the cameras obtain 75 points, which represent the $\mathrm{x}, \mathrm{y}$, and $\mathrm{z}$ coordinates of 25 body joints, 7 location independent features are extracted from them to reduce the computational load. These include the height,left hip angle, right hip angle, left knee angle, right knee angle, chest angle, and chest-knee angle. They were calculated from the body joints depth coordinates using cosine formula.

Table 1 shows the summary of results of machine learning and their performance using 10-fold cross validation. As shown in Table 1 the SVM outperforms other machine learning classifiers.

\begin{tabular}{|l|l|l|l|l|}
\hline Classifier & Accuracy & Recall & Precision & F1 score \\
\hline SVM(RBF) & $83.42 \%$ & 0.83 & 0.82 & 0.81 \\
\hline Naive Bayes & $74.56 \%$ & 0.74 & 0.73 & 0.74 \\
\hline Logistic Regression & $80.91 \%$ & 0.80 & 0.79 & 0.80 \\
\hline KNN & $76.98 \%$ & 0.76 & 0.75 & 076 \\
\hline Random Forests & $77.08 \%$ & 0.77 & 0.76 & 0.77 \\
\hline
\end{tabular}

Table 1. Machine Learning classifiers and their performance with 10 fold cross validation 
Table 2 shows the summary of results of deep learning and their performance using 10-fold cross validation. As shown in Table 2 the 1D-CNN outperforms other deep learning classifiers such as 2D-CNN, LSTM, BiLSTM.

\begin{tabular}{|l|l|l|l|l|}
\hline Classifier & Accuracy & Recall & Precision & F1 score \\
\hline MLP & $82.56 \%$ & 0.82 & 0.82 & 0.82 \\
\hline 1D-CNN & $93.45 \%$ & 0.93 & 0.92 & 0.93 \\
\hline 2D-CNN & $91.59 \%$ & 0.91 & 0.90 & 0.91 \\
\hline LSTM & $88.19 \%$ & 0.88 & 0.87 & 0.88 \\
\hline BiLSTM & $90.87 \%$ & 0.90 & 0.90 & 0.90 \\
\hline
\end{tabular}

Table 2. Deep Learning classifiers and their performance with 10-fold cross validation

Table 3 shows the summary of results of machine learning and their performance using 5 -fold cross validation. As shown in Table 3 the MLP outperforms other machine learning classifiers.

\begin{tabular}{|l|l|l|l|l|}
\hline Classifier & Accuracy & Precision & Recall & F-measure \\
\hline MLP & 81.56 & 0.81 & 0.8 & 0.81 \\
\hline SVM & 81.02 & 0.81 & 0.8 & 0.81 \\
\hline Naive Bayes & 71.36 & 0.71 & 0.7 & 0.71 \\
\hline Logistic Regression & 71.26 & 0.71 & 0.7 & 0.71 \\
\hline KNN & 74.87 & 0.75 & 0.74 & 75 \\
\hline Random Forest & 76.34 & 0.76 & 0.76 & 0.76 \\
\hline
\end{tabular}

Table 3. Machine Learning classifiers and their performance with 5-fold cross validation

Table 4 shows the summary of results of deep learning and their performance using 5 -fold cross validation. As shown in Table 4 the $1 \mathrm{D}$-CNN outperforms other deep learning classifiers.

\begin{tabular}{|l|l|l|l|l|}
\hline Classifier & Accuracy & Precision & Recall & F-measure \\
\hline 1D-CNN & 91.51 & 0.91 & 0.91 & 0.91 \\
\hline 2D-CNN & 90.96 & 0.91 & 0.9 & 0.91 \\
\hline LSTM & 89.56 & 0.89 & 0.88 & 0.89 \\
\hline BiLSTM & 88.98 & 0.89 & 0.88 & 0.89 \\
\hline
\end{tabular}

Table 4. Deep Learning classifiers and their performance with 5 -fold cross validation

Table 5 shows the summary of results of machine learning and their performance using 2-fold cross validation. As shown in Table 5 the SVM outperforms other machine learning classifiers. 


\begin{tabular}{|l|l|l|l|l|}
\hline Classifier & Accuracy & Precision & Recall & F-measure \\
\hline MLP & 80.42 & 0.8 & 0.79 & 0.8 \\
\hline SVM & 81.01 & 0.81 & 0.81 & 0.81 \\
\hline Na've Bayes & 70.94 & 0.7 & 0.7 & 0.7 \\
\hline Logistic Regression & 70.59 & 0.7 & 0.7 & 0.7 \\
\hline KNN & 74.29 & 0.74 & 0.74 & 74 \\
\hline Random Forest & 75.63 & 0.75 & 0.74 & 0.75 \\
\hline
\end{tabular}

Table 5. Machine Learning classifiers and their performance with 2-fold cross validation

Table 6 shows the summary of results of deep learning learning and their performance using 2-fold cross validation. As shown in Table 6 the 1D-CNN outperforms other deep learning classifiers.

\begin{tabular}{|l|l|l|l|l|}
\hline Classifier & Accuracy & Precision & Recall & F-measure \\
\hline 1D-CNN & 90.23 & 0.9 & 0.89 & 0.9 \\
\hline 2D-CNN & 89.67 & 0.89 & 0.88 & 0.89 \\
\hline LSTM & 88.19 & 0.88 & 0.87 & 0.88 \\
\hline BiLSTM & 87.76 & 0.87 & 0.86 & 0.87 \\
\hline
\end{tabular}

Table 6. Deep Learning classifiers and their performance with 2 -fold cross validation

\section{Conclusion}

The human posture detection is important in remote monitoring of patient. However, most of the current approaches cannot perfectly detect different human postures such as sitting, standing and lying down. Therefore, in this study, we proposed an approach based on the machine learning and deep learning approaches to detect different posture detection.

\section{Acknowledgement}

This work is supported in part by the Ajman University Internal Research Grant.

\section{References}

1. Ahsan Adeel, Mandar Gogate, and Amir Hussain. Contextual deep learning-based audio-visual switching for speech enhancement in real-world environments. Information Fusion, 59:163-170, 2020.

2. Rami Ahmed, Mandar Gogate, Ahsen Tahir, Kia Dashtipour, Bassam Al-Tamimi, Ahmad Hawalah, Mohammed A El-Affendi, and Amir Hussain. Deep neural network-based contextual recognition of arabic handwritten scripts. Entropy, $23(3): 340,2021$. 
3. H. Alaoui, M. T. Moutacalli, and M. Adda. Ai-enabled high-level layer for posture recognition using the azure kinect in unity3d. In 2020 IEEE 4th International Conference on Image Processing, Applications and Systems (IPAS), pages 155$161,2020$.

4. Abdulrahman S Alqarafi, Ahsan Adeel, Mandar Gogate, Kia Dashitpour, Amir Hussain, and Tariq Durrani. Toward's arabic multi-modal sentiment analysis. In International Conference in Communications, Signal Processing, and Systems, pages 2378-2386. Springer, 2017.

5. Syed Muhammad Asad, Shuja Ansari, Metin Ozturk, Rao Naveed Bin Rais, Kia Dashtipour, Sajjad Hussain, Qammer H Abbasi, and Muhammad Ali Imran. Mobility management-based autonomous energy-aware framework using machine learning approach in dense mobile networks. Signals, 1(2):170-187, 2020.

6. Syed Muhammad Asad, Kia Dashtipour, Sajjad Hussain, Qammer Hussain Abbasi, and Muhammad Ali Imran. Travelers-tracing and mobility profiling using machine learning in railway systems. In 2020 International Conference on UKChina Emerging Technologies (UCET), pages 1-4. IEEE, 2020.

7. Andrew Churcher, Rehmat Ullah, Jawad Ahmad, Fawad Masood, Mandar Gogate, Fehaid Alqahtani, Boubakr Nour, William J Buchanan, et al. An experimental analysis of attack classification using machine learning in iot networks. Sensors, 21(2):446, 2021.

8. Kia Dashtipour, Mandar Gogate, Ahsan Adeel, Abdulrahman Algarafi, Newton Howard, and Amir Hussain. Persian named entity recognition. In 2017 IEEE 16th International Conference on Cognitive Informatics $\&$ Cognitive Computing (ICCI* $C C)$, pages 79-83. IEEE, 2017.

9. Kia Dashtipour, Mandar Gogate, Ahsan Adeel, Amir Hussain, Abdulrahman Alqarafi, and Tariq Durrani. A comparative study of persian sentiment analysis based on different feature combinations. In International Conference in Communications, Signal Processing, and Systems, pages 2288-2294. Springer, 2017.

10. Kia Dashtipour, Mandar Gogate, Ahsan Adeel, Cosimo Ieracitano, Hadi Larijani, and Amir Hussain. Exploiting deep learning for persian sentiment analysis. In International conference on brain inspired cognitive systems, pages 597-604. Springer, 2018.

11. Kia Dashtipour, Mandar Gogate, Ahsan Adeel, Hadi Larijani, and Amir Hussain. Sentiment analysis of persian movie reviews using deep learning. Entropy, 23(5):596, 2021.

12. Kia Dashtipour, Mandar Gogate, Erik Cambria, and Amir Hussain. A novel context-aware multimodal framework for persian sentiment analysis. arXiv preprint arXiv:2103.02636, 2021.

13. Kia Dashtipour, Mandar Gogate, Jingpeng Li, Fengling Jiang, Bin Kong, and Amir Hussain. A hybrid persian sentiment analysis framework: Integrating dependency grammar based rules and deep neural networks. Neurocomputing, 380:1-10, 2020.

14. Kia Dashtipour, Amir Hussain, and Alexander Gelbukh. Adaptation of sentiment analysis techniques to persian language. In International Conference on Computational Linguistics and Intelligent Text Processing, pages 129-140. Springer, 2017.

15. Kia Dashtipour, Amir Hussain, Qiang Zhou, Alexander Gelbukh, Ahmad YA Hawalah, and Erik Cambria. Persent: a freely available persian sentiment lexicon. In International Conference on Brain Inspired Cognitive Systems, pages 310-320. Springer, 2016.

16. Kia Dashtipour, Soujanya Poria, Amir Hussain, Erik Cambria, Ahmad YA Hawalah, Alexander Gelbukh, and Qiang Zhou. Multilingual sentiment analysis: 
state of the art and independent comparison of techniques. Cognitive computation, 8(4):757-771, 2016.

17. Kia Dashtipour, Ali Raza, Alexander Gelbukh, Rui Zhang, Erik Cambria, and Amir Hussain. Persent 2.0: Persian sentiment lexicon enriched with domain-specific words. In International Conference on Brain Inspired Cognitive Systems, pages 497-509. Springer, 2019.

18. Kia Dashtipour, William Taylor, Shuja Ansari, Mandar Gogate, Adnan Zahid, Yusuf Sambo, Amir Hussain, Qammer Abbasi, and Muhammad Imran. Public perception towards fifth generation of cellular networks $(5 \mathrm{~g})$ on social media. Frontiers in Big Data, 2021.

19. Alexander RT Gepperth, Thomas Hecht, and Mandar Gogate. A generative learning approach to sensor fusion and change detection. Cognitive Computation, $8(5): 806-817,2016$.

20. S. Ghazal and U. S. Khan. Human posture classification using skeleton information. In 2018 International Conference on Computing, Mathematics and Engineering Technologies (iCoMET), pages 1-4, 2018.

21. Mandar Gogate, Ahsan Adeel, and Amir Hussain. Deep learning driven multimodal fusion for automated deception detection. In 2017 IEEE Symposium Series on Computational Intelligence (SSCI), pages 1-6. IEEE, 2017.

22. Mandar Gogate, Ahsan Adeel, and Amir Hussain. A novel brain-inspired compression-based optimised multimodal fusion for emotion recognition. In 2017 IEEE Symposium Series on Computational Intelligence (SSCI), pages 1-7. IEEE, 2017.

23. Mandar Gogate, Ahsan Adeel, Ricard Marxer, Jon Barker, and Amir Hussain. Dnn driven speaker independent audio-visual mask estimation for speech separation. arXiv preprint arXiv:1808.00060, 2018.

24. Mandar Gogate, Kia Dashtipour, Ahsan Adeel, and Amir Hussain. Cochleanet: A robust language-independent audio-visual model for real-time speech enhancement. Information Fusion, 63:273-285, 2020.

25. Mandar Gogate, Kia Dashtipour, and Amir Hussain. Visual speech in real noisy environments (vision): A novel benchmark dataset and deep learning-based baseline system. Proc. Interspeech 2020, pages 4521-4525, 2020.

26. Mandar Gogate, Amir Hussain, and Kaizhu Huang. Random features and random neurons for brain-inspired big data analytics. In 2019 International Conference on Data Mining Workshops (ICDMW), pages 522-529. IEEE, 2019.

27. Imane Guellil, Ahsan Adeel, Faical Azouaou, Fodil Benali, Ala-Eddine Hachani, Kia Dashtipour, Mandar Gogate, Cosimo Ieracitano, Reza Kashani, and Amir Hussain. A semi-supervised approach for sentiment analysis of arab (ic + izi) messages: Application to the algerian dialect. SN Computer Science, 2(2):1-18, 2021.

28. Zil E Huma, Shahid Latif, Jawad Ahmad, Zeba Idrees, Anas Ibrar, Zhuo Zou, Fehaid Alqahtani, and Fatmah Baothman. A hybrid deep random neural network for cyberattack detection in the industrial internet of things. IEEE Access, 9:55595$55605,2021$.

29. Amir Hussain, Ahsen Tahir, Zain Hussain, Zakariya Sheikh, Mandar Gogate, Kia Dashtipour, Azhar Ali, and Aziz Sheikh. Artificial intelligence-enabled analysis of uk and us public attitudes on facebook and twitter towards covid-19 vaccinations. medRxiv, 2020.

30. Intisar O Hussien, Kia Dashtipour, and Amir Hussain. Comparison of sentiment analysis approaches using modern arabic and sudanese dialect. In International Conference on Brain Inspired Cognitive Systems, pages 615-624. Springer, 2018. 
31. Cosimo Ieracitano, Ahsan Adeel, Mandar Gogate, Kia Dashtipour, Francesco Carlo Morabito, Hadi Larijani, Ali Raza, and Amir Hussain. Statistical analysis driven optimized deep learning system for intrusion detection. In International Conference on Brain Inspired Cognitive Systems, pages 759-769. Springer, 2018.

32. Fengling Jiang, Bin Kong, Jingpeng Li, Kia Dashtipour, and Mandar Gogate. Robust visual saliency optimization based on bidirectional markov chains. Cognitive Computation, pages 1-12, 2020.

33. Jaehyun Lee, Hyosung Joo, Junglyeon Lee, and Youngjoon Chee. Automatic classification of squat posture using inertial sensors: Deep learning approach. Sensors, 20(2), 2020.

34. Sidrah Liaqat, Kia Dashtipour, Kamran Arshad, Khaled Assaleh, and Naeem Ramzan. A hybrid posture detection framework: Integrating machine learning and deep neural networks. IEEE Sensors Journal, 21(7):9515-9522, 2021.

35. Sidrah Liaqat, Kia Dashtipour, Kamran Arshad, and Naeem Ramzan. Non invasive skin hydration level detection using machine learning. Electronics, 9(7):1086, 2020.

36. Sidrah Liaqat, Kia Dashtipour, Adnan Zahid, Khaled Assaleh, Kamran Arshad, and Naeem Ramzan. Detection of atrial fibrillation using a machine learning approach. Information, 11(12):549, 2020.

37. Congcong Ma, Wenfeng Li, Raffaele Gravina, and Giancarlo Fortino. Posture detection based on smart cushion for wheelchair users. Sensors, 17(4):719, 2017.

38. G. Matar, J. M. Lina, and G. Kaddoum. Artificial neural network for in-bed posture classification using bed-sheet pressure sensors. IEEE Journal of Biomedical and Health Informatics, 24(1):101-110, 2020.

39. Abozar Nasirahmadi, Barbara Sturm, Sandra Edwards, Knut-Håkan Jeppsson, Anne-Charlotte Olsson, Simone Müller, and Oliver Hensel. Deep learning and machine vision approaches for posture detection of individual pigs. Sensors, 19(17):3738, 2019.

40. Shibli Nisar, Muhammad Tariq, Ahsan Adeel, Mandar Gogate, and Amir Hussain. Cognitively inspired feature extraction and speech recognition for automated hearing loss testing. Cognitive Computation, 11(4):489-502, 2019.

41. Metin Ozturk, Mandar Gogate, Oluwakayode Onireti, Ahsan Adeel, Amir Hussain, and Muhammad A Imran. A novel deep learning driven, low-cost mobility prediction approach for $5 \mathrm{~g}$ cellular networks: The case of the control/data separation architecture (cdsa). Neurocomputing, 358:479-489, 2019.

42. L Panini and Rita Cucchiara. A machine learning approach for human posture detection in domotics applications. In 12th International Conference on Image Analysis and Processing, 2003. Proceedings., pages 103-108. IEEE, 2003.

43. Author1 Qassoud, Author2 Bolic, and Author3 Rajan. Posture-and-fall-detectionsystem-using-3d-motion-sensors, 2018.

44. Rui Sacchetti, Tiago Teixeira, Bruno Barbosa, AJ Neves, Sandra C Soares, and Isabel D Dimas. Human body posture detection in context: the case of teaching and learning environments. SIGNAL 2018 Editors, 87:79-84, 2018.

45. Ashraya Samba Shiva, Mandar Gogate, Newton Howard, Bruce Graham, and Amir Hussain. Complex-valued computational model of hippocampal ca3 recurrent collaterals. In 2017 IEEE 16th International Conference on Cognitive Informatics 86 Cognitive Computing (ICCI* CC), pages 161-166. IEEE, 2017.

46. William Taylor, Syed Aziz Shah, Kia Dashtipour, Adnan Zahid, Qammer H Abbasi, and Muhammad Ali Imran. An intelligent non-invasive real-time human activity recognition system for next-generation healthcare. Sensors, 20(9):2653, 2020. 
47. Zheqi Yu, Pedro Machado, Adnan Zahid, Amir M Abdulghani, Kia Dashtipour, Hadi Heidari, Muhammad A Imran, and Qammer H Abbasi. Energy and performance trade-off optimization in heterogeneous computing via reinforcement learning. Electronics, 9(11):1812, 2020. 